\title{
Intractable Hiccup Due to Multiple Sclerosis: MR Imaging of Medullary Plaque
}

\author{
Yung-Yee Chang, Hsiu-Shen Wu, Tsung-Chia Tsai and Jia-Shou Liu
}

\begin{abstract}
We describe a 31-year-old man with clinically definite multiple sclerosis who presented with intractable hiccup for one month prior to admission. The responsible lesion was confirmed by magnetic resonance imaging to be a plaque in the ventral paramedian portion of the medulla oblongata.

Résumé: Hoquet incoercible dû à une sclérose en plaques: visualisation d'une plaque médullaire par RMN. Nous décrivons le cas d'un homme de 31 ans, atteint d'une sclérose en plaques cliniquement certaine, qui a présenté un hoquet incoercible un mois avant son admission. L'imagerie par résonance magnétique a confirmé que la lésion en cause était une plaque située dans la portion paramédiane ventrale du bulbe rachidien.
\end{abstract}

Can. J. Neurol. Sci. 1994; 21: 271-272

Although multiple sclerosis (MS) is characterized by protean manifestations, intractable hiccup (IH) is rare and has been described only in isolated case reports. ${ }^{1-3}$ We describe a patient with clinically definite MS presenting with IH who responded well to corticosteroid therapy.

\section{Case Report}

A 31 -year-old businessman experienced $\mathrm{IH}$, vertigo and vomiting for two weeks in December 1987. Six weeks later, the patient developed transient episodes of double vision on left gaze and paroxysmal attacks of left facial numbness. Cranial nerve examination disclosed impaired left visual acuity, horizontal nystagmus on left gaze, hypesthesia and dysesthesia in the left face and ipsilateral neurosensory hearing impairment. Additional neurologic findings were mild paraparesis with brisk tendon reflexes, right dysmetria and symmetrical sensory disturbance below the $T_{10}$ level bilaterally.

Laboratory examinations were unremarkable, except that cerebrospinal fluid (CSF) study showed a slight mononuclear pleocytosis as well as mild protein elevation with $18 \%$ IgG. Pattern-shift visualevoked potentials (EP) revealed prolonged latencies bilaterally. Studies of short-latency somatosensory EP and brainstem auditory EP were compatible with intrinsic brainstem lesions. However, a cranial computed tomographic (CT) scan was unremarkable. In the following 4 years, he had one relapse and remission of the right hemiparesis and brachial monoparesthesia.

He was readmitted to our hospital in September 1992 because of $\mathrm{IH}$ for about one month. The patient had 10 to 15 episodes of hiccup per day and each episode lasted minutes to hours. The hiccups could not be suppressed by Valsalva maneuver, vagal stimulation, or irritation of the uvula. Therapeutic trials with metoclopramide, chlorpromazine and diphenylhydantoin were all in vain. The patient also complained of episodic vomiting, easy choking, double vision, unsteady gait and a symmetrical ascending numbness and tight sensation up to the occipital region.

On neurologic examination, slow saccadic movement on right gaze was present with concomitant horizontal nystagmus. Dysarthria, bilateral trigeminal hypesthesia, cerebellar dysmetria and diminished proprioception of all extremities were also noted. There was no motor weakness, but a generalized hyperreflexia was identified. A cranial CT scan showed no discernible abnormality. Magnetic resonance imaging (MRI) with T1-weighted sagittal images demonstrated hypo-intense paramedian lesions along the ventral medulla. Axial views showed several plaques in the rostral and medial portion of the medulla oblongata. The neighboring paramedian plaques straddled the midline portion to form a large bilateral plaque (Figure 1A, B). The plaque involved bilateral pyramidal tracts, medial lemnisci, olives and paramedian reticular formations. On prednisone $(60 \mathrm{mg} /$ day) and metoclopramide, the hiccups resolved within one week, and his speech, sensory and cerebellar dysfunction all improved. His condition has been stable since then.

\section{Discussion}

Newsom-Davis described hiccup as being controlled by a suprasegmental mechanism entirely independent of the respiratory system. ${ }^{4}$ IH caused by brainstem dysfunction has been attributed to the lesions in the hiccup reflex arc entailing the following structures: the dorsal portion of the nucleus tractus solitarius (inspiratory center), the ventral group of nucleus ambiguus (expiratory center), pneumotaxic center, and hypothetical apneustic center. ${ }^{3.5-8}$ The MS plaques in the ventral medulla of our patient suggest that they may potentially perturb the neighboring hiccup reflex arc or interfere with the descending inhibitory cortico-medullary pathways. ${ }^{9}$

From the Deparment of Neurology (Y.-Y.C., J.-S.L.) and Radiology (T.-C.T.), Kaohsiung Medical College and Department of Neurology (H.-S.W.). Chang Gung Memorial Hospital, Kaohsiung, Taiwan.

RECEIVED SEPTEMBER 27, 1993. ACCEPTED IN FINAL FORM JANUARY 14, 1994. Reprint requests to: Dr. J.S. Liu, Department of Neurology, Kaohsiung Medical College, No. 100, Shih-Chuan Ist Road, Kaohsiung 807, Taiwan. 


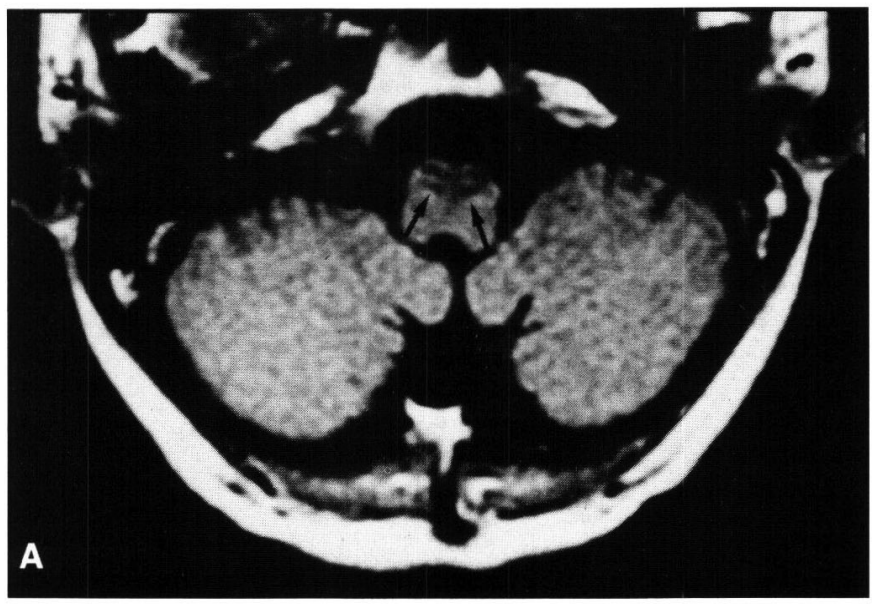

Figure 1A. TI-weighted axial MRI showing a fan-shape plaque (arrows) in the paramedian ventral medulla at the level of vagus nuclei.$(T R=600 \mathrm{msec}, T E=20 \mathrm{msec})$.

The concept of $\mathrm{IH}$ as a dysfunction of hiccup reflex arc indicates that we should search cautiously for underlying hazardous and/or treatable condition. Howard et al. reported two MS patients with insidious onset of hiccups led to respiratory failure. ${ }^{10}$ We stress that an early diagnosis may prevent a potential catastrophe.

\section{ACKNOWLEDGEMENT}

We wish to thank Mrs Sandrine Tsai for her preparation of the manuscript.

\section{REFERENCES}

1. McFarling DA, Susac JO. Hoquet diabolique: intractable hiccups as a manifestation of multiple sclerosis. Neurology 1979; 29: 797-801.

2. Jacobson PL, Messenheimer JA, Farmer TW. Treatment of intractable hiccups with valproic acid. Neurology 1981; 31 : 1458-1460.

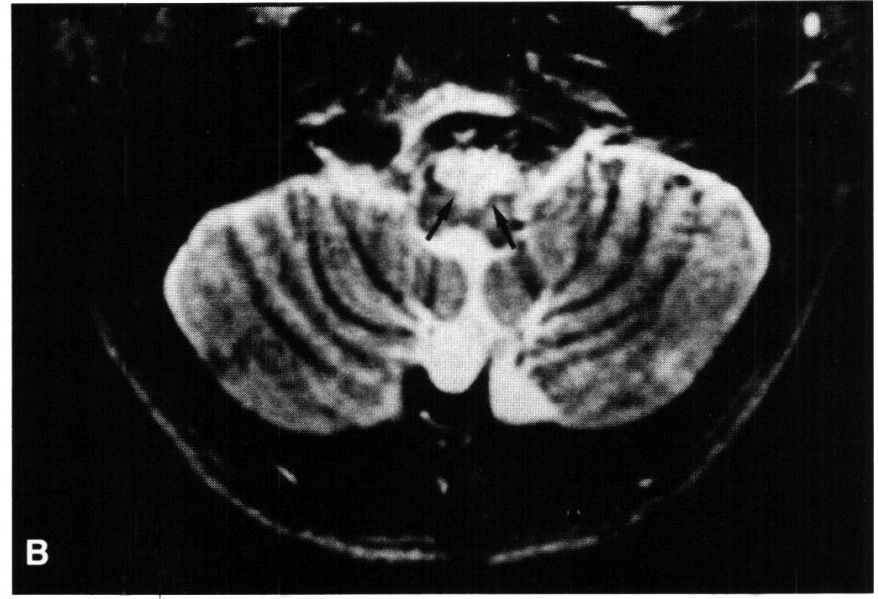

Figure 1B. T2-weighted spin-echo image at the same level shows a prolonged $T 2$ lesion in the ventromedial medulla. $(T R=2000 \mathrm{msec}$, $T E=90 \mathrm{msec}$ )

3. Birkhead R, Friedman JH. Hiccups and vomiting as initial manifestation of multiple sclerosis. J Neurol Neurosurg Psychiatry 1987; 50: 232-233.

4. Newsom-Davis J. An experimental study of hiccup. Brain 1970; 93: 851-872.

5. Al Deeb SM, Sharif H, Al Moutaery K, Biary N. Intractable hiccup induced by brainstem lesion. J Neurol Sci 1991; 103: 144-150.

6. Currier RD, Giles CL, DeJong RN. Some comments on Wallenberg lateral medullary syndrome. Neurology 1961; 11: 778-791.

7. Stotka VL, Barcay SJ, Bell HS, Clare FB. Intractable hiccup as the primary manifestation of brain stem tumor. Am J Med 1962;32: 313-315.

8. Kozik M, Owsianowska L. Persistent hiccoughs as the predominant symptom with a tumour of the medulla oblongata. J Neurol $1976 ; 212: 91-93$.

9. Brodal A. Neurological Anatomy: in Relation to Clinical Medicine, 3rd ed. Oxford: Oxford University Press. 1981; 394447.

10. Howard RS, Wiles CM, Hirsch NP, et al. Respiratory involvement in multiple sclerosis. Brain 1992; 115: 479-494. 